\title{
The ORFEUS II Echelle spectrum of HD 93521: A reference for interstellar molecular hydrogen
}

\author{
J. Barnstedt ${ }^{1}$, W. Gringel ${ }^{1}$, N. Kappelmann ${ }^{1}$, and M. Grewing ${ }^{1,2}$ \\ 1 Institut für Astronomie und Astrophysik, Abt. Astronomie, Eberhard-Karls-Universität Tübingen, Waldhäuserstr. 64, \\ D-72076 Tübingen, Germany \\ 2 Institut de Radio Astronomie Millimétrique (IRAM), 300 rue de la Piscine, F-38406 Saint Martin d'Hères, France
}

Received December 13, 1999; accepted January 21, 2000

\begin{abstract}
During the second flight of the ORFEUS-SPAS mission in November/December 1996, the Echelle spectrometer was used extensively by the Principal and Guest Investigator teams as one of the two focal plane instruments of the ORFEUS telescope. The spectrum of HD 93521 was obtained during this mission with a total integration time of $1740 \mathrm{~s}$. This spectrum shows numerous sharp interstellar absorption lines. We identified 198 lines of molecular hydrogen including at least 7 lines with a high velocity component. Also most of the 67 identified interstellar metal lines are visible with a high velocity component.

We present plots of the complete ORFEUS II Echelle spectrum together with tables of all identified interstellar absorption lines including all 14 detectable H I lines. In addition several identified stellar lines, partially with narrow absorption components, and stellar wind lines are given in a separate table.
\end{abstract}

Key words: stars: individual: HD 93521 - ISM: lines and bands - ISM: molecules - ultraviolet: ISM ultraviolet: stars

\section{Introduction}

The star HD 93521 is a high galactic latitude O-star. It has been used for many years as a tracer of the interstellar gas in the galactic halo, for which it is well suited due to its brightness $(V=7.04)$, high galactic latitude $\left(l=183^{\circ}, b=62^{\circ}\right)$ and large rotational velocity $\left(v \sin i \approx 400 \mathrm{~km} \mathrm{~s}^{-1}\right.$, Lennon et al. 1991). Together with a $z$-distance of about $1.5 \mathrm{kpc}$ (Irvine 1989), HD 93521 is an ideal candidate for studying kinematics of the halo gas,

Send offprint requests to: J. Barnstedt

e-mail: barnstedt@astro.uni-tuebingen.de since galactic rotation effects should be very small (Spitzer \& Fitzpatrick 1992).

With the Echelle spectrometer of the ORFEUS telescope (Orbiting and Retrievable Far and Extreme Ultraviolet Spectrometer) it was for the first time possible to observe all available absorption lines of interstellar molecular hydrogen towards HD 93521. In the gathered spectra we identified $198 \mathrm{H}_{2}$-lines. These lines are very narrow and strong but unsaturated, which was the reason why many of them were used for wavelength calibration of the ORFEUS Echelle spectrometer. Also 67 other interstellar absorption lines and 14 lines of the Lyman series were identified. For completeness stellar and stellar wind absorption lines are shown too.

We present the whole Echelle spectrum of HD 93521, obtained during the second ORFEUS-SPAS mission in November/December 1996. This spectrum has a good signal to noise ratio and the spectral resolution achieved is somewhat higher than the claimed resolution of $\lambda / \Delta \lambda=10.000$ (Barnstedt et al. 1999).

The plots presented in the Appendix show one Echelle order per plot for wavelengths above $1130 \AA$ (Echelle orders 40 to 49 ), and half an Echelle order per plot for Echelle orders 50 to $61(\lambda<1130 \AA)$ where all $\mathrm{H}_{2}$-lines are included.

\section{Data reduction and line identification}

Two separate observations of HD 93521 were obtained during two successive orbits with a total integration time of $1740 \mathrm{~s}$ (ORFEUS observation IDs 2276_2 and 2276_3, observation date: day 333 of 1996, GMT 04:56:05 - 05:14:05 and GMT 06:28:05 - 06:39:05). The two echelle images were coadded and then the standard extraction procedure was applied (Barnstedt et al. 1999) without any smoothing. An additional radial velocity correction of $-10 \mathrm{~km} \mathrm{~s}^{-1}$ was applied, which corrects the wavelength scale for the fact that the star was not 
absolutely centered in the entrance diaphragm of the telescope. The maximum uncertainty due to the $20^{\prime \prime}$ diameter of the diaphragm was $\pm 36 \mathrm{kms}^{-1}$, so the deviation of $-10 \mathrm{~km} \mathrm{~s}^{-1}$ corresponds to a pointing offset of $3^{\prime \prime}$, which is an excellent value for the ASTRO-SPAS satellite. The value of $-10 \mathrm{kms}^{-1}$ was estimated by comparing the observed radial velocity components with those already published (Spitzer \& Fitzpatrick 1992). The wavelength scale is heliocentric, a LSR scale would require an additional correction of $-1.6 \mathrm{~km} \mathrm{~s}^{-1}$, which is negligible.

As with all echelle spectra, the signal to noise ratio is best in the centre of the echelle orders, while it is reduced by a factor of about 1.4 at both ends of each order. Due to the blaze curve being not fully centered, the signal to noise ratio at the short wavelength end is significantly better than at the long wavelength end of each echelle order. There is also a slight deviation in the wavelength calibration at the short wavelength end of each order, which affects a wavelength range of about $5 \%$ in each order.

For line identifications we used the following line catalogues:

1. $\mathrm{H}_{2}$-lines:

- Morton \& Dinerstein (1976), except L10P1;

- Abgrall et al. (1993), correct wavelength for L10P1: $982.834 \AA$.

2. Metal lines and $\mathrm{HI}$ :

- Morton (1991);

- Kurucz CD No. 23, web-page (Kurucz 1995);

- Kelly (1968; and web-page);

- Feibelman \& Johannson (1995).

Identified lines from IUE spectra of HD 93521 above $1170 \AA$ are listed by Ramella et al. (1980), with exception of two interstellar lines: $\lambda 1260.4$, which is Si II and not Si III, and $\lambda 1304.4$, which is also Si II and not O I.

The tables and plots show blended lines also, for which a non-ambiguous identification or estimate of the intensity is not possible.

Lines with a lower energy level greater than zero are marked with an asterisk, ${ }^{*}$. All stellar lines are marked with a bracketed asterisk, $(*)$, and stellar wind lines are marked as (w) in the plots as well as in the tables.

\section{Discussion of spectral features}

The interstellar, stellar and wind absorption lines are visible in several or different radial velocity components. We therefore list and describe all occuring radial velocity components in Table 1.

We present tables of identified interstellar and stellar absorption lines. These tables show a running number for identification of the lines in the plots shown in the Appendix, the vacuum wavelength, the $\log (g f)$-value, the number of the radial velocity component (VC) applied as given in Table 1, and some remarks or the transition for the $\mathrm{H}_{2}$-lines. We will present and discuss the tables of molecular hydrogen lines, other interstellar lines, Lyman series lines and stellar absorption lines.

\subsection{Radial velocity components}

Table 1 lists 7 components of radial velocities used to identify absorption lines and features in the spectrum. The first two components are the interstellar absorptions at $-12 \mathrm{~km} \mathrm{~s}^{-1}$ and $-60 \mathrm{~km} \mathrm{~s}^{-1}$, which are the two strongest of well known interstellar components (Grewing et al. 1978; Keenan et al. 1995; Spitzer \& Fitzpatrick 1992, 1993). No. 3 gives the published value of the radial velocity of HD 93521 of $-16 \mathrm{~km} \mathrm{~s}^{-1}($ SIMBAD). No. 4 is the velocity of the emission of the geocoronal Ly- $\alpha$ line. This emission line results from a completely illuminated entrance aperture of the Echelle spectrometer which had a projected diameter of $20^{\prime \prime}$. The velocity of $36.5 \mathrm{~km} \mathrm{~s}^{-1}$ is the negative sum of two wavelength corrections applied to this spectrum: the heliocentric correction and the decentering correction $\left(26.5 \mathrm{~km} \mathrm{~s}^{-1}+10 \mathrm{~km} \mathrm{~s}^{-1}\right)$.

Some stellar absorption lines show narrow absorption components resulting from winds, which have been observed previously (Bjorkman et al. 1994), but which are varying in time. We have identified two such components in serveral lines and they are listed as numbers 5 and 6 in Table 1. Component 7 represents the radial velocities of the strong Si III $\lambda \lambda 1300$ triplets, which are also due to stellar wind absorption (Massa 1995).

Table 1. Table of radial velocity components (VC)

\begin{tabular}{rrl}
\hline $\begin{array}{r}\text { VC } \\
\text { No. }\end{array}$ & $\begin{array}{r}\text { Rad.vel. } \\
{\left[\mathrm{km} \mathrm{s}^{-1}\right]}\end{array}$ & Description \\
\hline 1 & -12 & first (main) interstellar component \\
2 & -60 & high velocity interstellar component \\
3 & -16 & radial velocity of HD 93521 \\
4 & 36.5 & geocoronal Ly- $\alpha$ emission \\
5 & -270 & 1. wind feature in stellar absorption lines \\
6 & -340 & 2. wind feature in stellar absorption lines \\
7 & -80 & wind feature in Si III triplets \\
\hline
\end{tabular}

\subsection{Interstellar molecular hydrogen}

For most of the $\mathrm{H}_{2}$-lines only the main velocity component No. 1 was observed, but for some unblended lines the high velocity component could be seen also. A detailed discussion of column densities and curve of growths will be published in a separate paper (Gringel et al., in preparation).

Previous Copernicus measurements of selected $\mathrm{H}_{2}-$ lines only led to an upper limit of $\log N\left(\mathrm{H}_{2}\right)<18.54$ (Savage et al. 1992). The high velocity components were not detected by Copernicus. 
Table 2. Table of identified or possible interstellar molecular hydrogen lines. $\mathrm{VC}$ is the velocity component as given in Table 1

\begin{tabular}{rrrlll}
\hline No. & $\lambda[\AA]$ & $\log (f)$ & VC & Transition & Remarks \\
\hline 1 & 918.411 & -2.795 & 1 & L18P1 & blend \\
2 & 918.427 & -1.889 & 1 & W5Q3 & blend \\
3 & 919.410 & -2.430 & 1 & L18R2 & blend \\
4 & 919.545 & -2.345 & 1 & W5P3 & blend \\
5 & 920.242 & -2.767 & 1 & L18P2 & \\
6 & 924.643 & -2.412 & 1 & L17R1 & \\
7 & 925.173 & -2.707 & 1 & L17P1 & \\
8 & 927.020 & -2.628 & 1 & L17P2 & \\
9 & 928.437 & -2.548 & 1 & L17R3 & \\
10 & 929.534 & -1.470 & 1 & W4R0 & blend \\
11 & 929.687 & -1.810 & 1 & W4R1 & blend \\
12 & 929.688 & -2.595 & 1 & L17P3 & blend \\
13 & 931.063 & -1.991 & 1 & L16R0 & \\
14 & 931.732 & -2.151 & 1 & L16R1 & blend \\
15 & 931.779 & -1.714 & 1 & W4Q2 & blend \\
16 & 931.811 & -1.979 & 1 & W4R3 & blend \\
17 & 932.270 & -2.621 & 1 & L16P1 & \\
18 & 932.606 & -2.318 & 1 & W4P2 & \\
19 & 933.185 & -2.563 & 1 & L17P4 & blend \\
20 & 933.243 & -2.202 & 1 & L16R2 & blend \\
21 & 933.581 & -1.714 & 1 & W4Q3 & \\
22 & 934.146 & -2.643 & 1 & L16P2 & \\
23 & 934.789 & -2.145 & 1 & W4P3 & blend \\
24 & 935.537 & -2.498 & 1 & L17R5 & blend \\
25 & 935.578 & -2.236 & 1 & L16R3 & \\
26 & 935.959 & -1.717 & 1 & W4Q4 & \\
27 & 936.859 & -2.679 & 1 & L16P3 & \\
28 & 938.468 & -2.036 & 1 & L15R0 & \\
29 & 939.124 & -2.207 & 1 & L15R1 & \\
30 & 939.710 & -2.536 & 1 & L15P1 & \\
31 & 940.627 & -2.256 & 1 & L15R2 & \\
32 & 941.601 & -2.471 & 1 & L15P2 & \\
33 & 942.966 & -2.293 & 1 & L15R3 & \\
34 & 944.331 & -2.451 & 1 & L15P3 & \\
35 & 946.129 & -2.342 & 1 & L15R4 & blend \\
36 & 946.170 & -2.958 & 1 & L14R0 & blend \\
37 & 946.386 & -1.889 & 1 & W3R1 & blend \\
38 & 946.425 & -1.207 & 1 & W3R0 & blend \\
\hline & & & & & \\
\hline
\end{tabular}

\subsection{Interstellar metal lines}

Most of the metal lines can be observed in both interstellar components with the second component being only slightly weaker than the main component. High resolution spectra do show more components (Spitzer \& Fitzpatrick 1993), but in the ORFEUS echelle spectra only two well separated components are seen. The separation is best seen in the ArI lines $\lambda 1048$ and $\lambda 1067$ and the NI triplett $\lambda 1134$. Interstellar O VI at $\lambda 1032$ and $\lambda 1037$ appears quite broad. Widmann estimated a $N(\mathrm{O}$ VI $)=(0.99 \pm 0.15) 10^{14} \mathrm{~cm}^{-2}$ from these ORFEUS echelle spectra (Widmann et al. 1998; Widmann 1999).
Table 2. continued

\begin{tabular}{|c|c|c|c|c|c|}
\hline No. & $\lambda[\AA]$ & $\log (f)$ & $\mathrm{VC}$ & Transition & Remarks \\
\hline 39 & 946.986 & -2.728 & 1 & L14R1 & blend \\
\hline 40 & 947.113 & -1.876 & 1 & W3R2 & \\
\hline 41 & 947.425 & -1.564 & 1 & W3Q1 & \\
\hline 42 & 947.517 & -2.454 & 1 & L14P1 & \\
\hline 43 & 948.418 & -1.893 & 1 & W3R3 & blend \\
\hline 44 & 948.472 & -1.917 & 1 & L14R2 & blend \\
\hline 45 & 948.618 & -1.564 & 1 & W3Q2 & blend \\
\hline 46 & 949.355 & -2.041 & 1 & L14P2 & blend \\
\hline 47 & 950.316 & -1.903 & 1 & W3R4 & blend \\
\hline 48 & 950.401 & -1.564 & 1 & W3Q3 & blend \\
\hline 49 & 950.820 & -2.003 & 1 & L14R3 & blend \\
\hline 50 & 951.672 & -1.896 & 1 & W3P3 & \\
\hline 51 & 952.256 & -2.416 & 1 & L15P5 & blend \\
\hline 52 & 952.276 & -3.151 & 1 & L14P3 & blend \\
\hline 53 & 952.758 & -1.565 & 1 & W3Q4 & blend \\
\hline 54 & 952.807 & -1.762 & 1 & W3R5 & blend \\
\hline 55 & 954.419 & -1.860 & 1 & L13R0 & blend \\
\hline 56 & 954.475 & -1.873 & 1 & W3P4 & blend \\
\hline 57 & 955.067 & -2.024 & 1 & L13R1 & \\
\hline 58 & 955.682 & -1.567 & 1 & W3Q5 & \\
\hline 59 & 955.711 & -2.374 & 1 & L13P1 & \\
\hline 60 & 956.581 & -2.064 & 1 & L13R2 & \\
\hline 61 & 957.654 & -2.316 & 1 & L13P2 & \\
\hline 62 & 958.949 & -2.087 & 1 & L13R3 & \\
\hline 63 & 960.452 & -2.307 & 1 & L13P3 & \\
\hline 64 & 962.978 & -1.889 & 1 & $\mathrm{~L} 12 \mathrm{R} 0$ & \\
\hline 65 & 963.609 & -2.163 & 1 & L12R1 & blend \\
\hline 66 & 964.312 & -2.300 & 1 & L12P1 & \\
\hline 67 & 964.988 & -2.821 & 1 & $\mathrm{~L} 12 \mathrm{R} 2$ & blend \\
\hline 68 & 964.988 & -1.162 & 1 & W2R0 & blend \\
\hline 69 & 965.067 & -1.453 & 1 & W2R1 & blend \\
\hline 70 & 965.793 & -1.491 & 1 & W2R2 & \\
\hline 71 & 966.097 & -1.457 & 1 & W2Q1 & \\
\hline 72 & 966.272 & -2.189 & 1 & $\mathrm{~L} 12 \mathrm{P} 2$ & \\
\hline 73 & 966.780 & -2.104 & 1 & W2R3 & \\
\hline 74 & 967.278 & -1.458 & 1 & W2Q2 & \\
\hline 75 & 967.674 & -1.667 & 1 & L12R3 & \\
\hline 76 & 968.293 & -2.148 & 1 & $\mathrm{~W} 2 \mathrm{P} 2$ & \\
\hline 77 & 969.047 & -1.458 & 1 & W2Q3 & blend \\
\hline 78 & 969.086 & -2.095 & 1 & L12P3 & blend \\
\hline 79 & 970.560 & -2.022 & 1 & W2P3 & \\
\hline 80 & 971.386 & -1.460 & 1 & W2Q4 & \\
\hline 81 & 971.984 & -1.705 & 1 & L11R0 & weak \\
\hline 82 & 973.348 & -2.231 & 1 & L11P1 & \\
\hline 83 & 974.156 & -1.903 & 1 & L11R2 & \\
\hline 84 & 975.343 & -2.178 & 1 & L11P2 & weak \\
\hline 85 & 978.216 & -2.175 & 1 & L11P3 & \\
\hline 86 & 981.441 & -1.690 & 1 & L10R0 & \\
\hline 87 & 982.074 & -1.876 & 1 & L10R1 & \\
\hline 88 & 982.834 & -2.169 & 1 & L10P1 & \\
\hline 89 & 983.595 & -1.950 & 1 & L10R2 & \\
\hline 90 & 984.866 & -2.091 & 1 & L10P2 & \\
\hline 91 & 985.632 & -1.157 & 1,2 & W1R0 & blend \\
\hline 92 & 985.651 & -1.474 & 1,2 & W1R1 & blend \\
\hline
\end{tabular}


Table 2. continued

Table 2. continued

\begin{tabular}{|c|c|c|c|c|c|}
\hline No. & $\lambda[\AA]$ & $\log (f)$ & $\mathrm{VC}$ & Transition & Remarks \\
\hline 93 & 985.967 & -2.108 & 1 & L10R3 & \\
\hline 94 & 986.246 & -1.570 & 1 & W1R2 & \\
\hline 95 & 986.798 & -1.439 & 1,2 & W1Q1 & \\
\hline 96 & 987.450 & -1.578 & 1 & W1R3 & \\
\hline 97 & 987.770 & -2.056 & 1 & L10P3 & \\
\hline 98 & 987.978 & -1.440 & 1 & W1Q2 & \\
\hline 99 & 991.388 & -1.939 & 1 & W1P3 & blend \\
\hline 100 & 991.394 & -1.587 & 1 & L9R0 & blend \\
\hline 101 & 992.018 & -1.441 & 1 & W1Q4 & blend \\
\hline 102 & 992.022 & -1.745 & 1 & L9R1 & blend \\
\hline 103 & 992.052 & -1.638 & 1 & W1R5 & blend \\
\hline 104 & 992.813 & -2.116 & 1 & L9P1 & \\
\hline 105 & 993.492 & -1.959 & 1 & L10R5 & blend, weak \\
\hline 106 & 993.549 & -1.780 & 1 & L9R2 & blend \\
\hline 107 & 994.229 & -1.885 & 1 & W1P4 & \\
\hline 108 & 994.876 & -2.066 & 1 & L9P2 & blend \\
\hline 109 & 994.924 & -1.442 & 1 & W1Q5 & blend \\
\hline 110 & 995.975 & -1.793 & 1 & L9R3 & \\
\hline 111 & 997.829 & -2.063 & 1,2 & L9P3 & see Add. \\
\hline 112 & 1001.826 & -1.575 & 1 & L8R0 & \\
\hline 113 & 1002.457 & -1.742 & 1,2 & L8R1 & \\
\hline 114 & 1003.304 & -2.076 & 1 & L8P1 & \\
\hline 115 & 1003.989 & -1.785 & 1 & L8R2 & \\
\hline 116 & 1005.397 & -2.009 & 1 & L8P2 & \\
\hline 117 & 1006.346 & -2.080 & 1 & L9P5 & blend \\
\hline 118 & 1006.418 & -1.812 & 1 & L8R3 & blend \\
\hline 119 & 1008.392 & -1.991 & 1 & L8P3 & blend \\
\hline 120 & 1008.502 & -1.672 & 1 & W0R1 & blend \\
\hline 121 & 1008.553 & -1.349 & 1 & WORO & blend \\
\hline 122 & 1009.030 & -1.783 & 1 & W0R2 & \\
\hline 123 & 1009.721 & -2.866 & 1 & L8R4 & blend \\
\hline 124 & 1009.772 & -1.623 & 1,2 & W0Q1 & blend \\
\hline 125 & 1010.132 & -1.833 & 1 & W0R3 & \\
\hline 126 & 1010.941 & -1.623 & 1 & W0Q2 & \\
\hline 127 & 1012.173 & -2.276 & 1 & W0P2 & blend \\
\hline 128 & 1012.261 & -1.983 & 1 & $\mathrm{~L} 8 \mathrm{P} 4$ & blend \\
\hline 129 & 1012.681 & -1.625 & 1 & W0Q3 & \\
\hline 130 & 1012.822 & -1.527 & 1 & L7R0 & \\
\hline 131 & 1013.434 & -1.688 & 1,2 & L7R1 & blend \\
\hline 132 & 1013.480 & -1.821 & 1 & W0R5 & blend \\
\hline 133 & 1014.334 & -2.051 & 1 & L7P1 & \\
\hline 134 & 1014.509 & -2.107 & 1 & W0P3 & \\
\hline 135 & 1014.977 & -1.724 & 1 & L7R2 & blend \\
\hline 136 & 1014.980 & -1.625 & 1 & W0Q4 & blend \\
\hline 137 & 1016.472 & -1.996 & 1 & L7P2 & \\
\hline 138 & 1017.390 & -2.036 & 1 & WOP4 & blend \\
\hline 139 & 1017.428 & -1.735 & 1 & L7R3 & blend \\
\hline 140 & 1019.506 & -1.991 & 1 & L7P3 & \\
\hline 141 & 1023.443 & -1.996 & 1 & L7P4 & \\
\hline 142 & 1024.364 & -1.540 & 1 & L6R0 & \\
\hline 143 & 1024.986 & -1.703 & 1 & $\mathrm{~L} 6 \mathrm{R} 1$ & blend \\
\hline 144 & 1024.991 & -1.842 & 1 & L7R5 & blend \\
\hline 145 & 1026.532 & -1.740 & 1 & L6R2 & \\
\hline 146 & 1028.103 & -1.983 & 1 & $\mathrm{~L} 6 \mathrm{P} 2$ & \\
\hline
\end{tabular}

\begin{tabular}{|c|c|c|c|c|c|}
\hline No. & $\lambda[\AA]$ & $\log (f)$ & $\mathrm{VC}$ & Transition & Remarks \\
\hline 147 & 1028.986 & -1.752 & 1 & L6R3 & \\
\hline 148 & 1031.191 & -1.966 & 1 & L6P3 & \\
\hline 149 & 1032.356 & -1.757 & 1 & L6R4 & \\
\hline 150 & 1036.546 & -1.567 & 1 & L5R0 & blend \\
\hline 151 & 1036.546 & -1.836 & 1 & L6R5 & blend \\
\hline 152 & 1037.146 & -1.733 & 1 & L5R1 & blend \\
\hline 153 & 1038.156 & -2.068 & 1 & L5P1 & \\
\hline 154 & 1038.690 & -1.770 & 1 & L5R2 & \\
\hline 155 & 1040.367 & -2.001 & 1 & $\mathrm{~L} 5 \mathrm{P} 2$ & \\
\hline 156 & 1041.156 & -1.785 & 1 & L5R3 & \\
\hline 157 & 1043.498 & -1.983 & 1 & L5P3 & \\
\hline 158 & 1044.546 & -1.791 & 1 & L5R4 & weak \\
\hline 159 & 1047.554 & -1.979 & 1 & L5P4 & \\
\hline 160 & 1049.366 & -1.629 & 1 & L4R0 & \\
\hline 161 & 1049.958 & -1.796 & 1 & L4R1 & \\
\hline 162 & 1051.031 & -2.125 & 1 & L4P1 & \\
\hline 163 & 1051.497 & -1.833 & 1 & $\mathrm{~L} 4 \mathrm{R} 2$ & \\
\hline 164 & 1053.281 & -2.056 & 1 & $\mathrm{~L} 4 \mathrm{P} 2$ & \\
\hline 165 & 1053.976 & -1.848 & 1 & L4R3 & \\
\hline 166 & 1056.469 & -2.037 & 1 & L4P3 & \\
\hline 167 & 1057.379 & -1.854 & 1 & $\mathrm{~L} 4 \mathrm{R} 4$ & weak \\
\hline 168 & 1060.580 & -2.032 & 1 & $\mathrm{~L} 4 \mathrm{P} 4$ & weak \\
\hline 169 & 1062.883 & -1.740 & 1 & L3R0 & blend \\
\hline 170 & 1063.460 & -1.907 & 1 & L3R1 & \\
\hline 171 & 1064.606 & -2.234 & 1 & L3P1 & \\
\hline 172 & 1064.995 & -1.947 & 1 & L3R2 & \\
\hline 173 & 1066.900 & -2.163 & 1 & L3P2 & \\
\hline 174 & 1067.478 & -1.963 & 1 & L3R3 & \\
\hline 175 & 1070.142 & -2.142 & 1 & L3P3 & \\
\hline 176 & 1077.138 & -1.924 & 1 & $\mathrm{~L} 2 \mathrm{R} 0$ & \\
\hline 177 & 1077.698 & -2.092 & 1 & L2R1 & \\
\hline 178 & 1078.925 & -2.415 & 1 & $\mathrm{~L} 2 \mathrm{P} 1$ & \\
\hline 179 & 1079.226 & -2.132 & 1 & L2R2 & \\
\hline 180 & 1081.265 & -2.344 & 1 & $\mathrm{~L} 2 \mathrm{P} 2$ & \\
\hline 181 & 1081.710 & -2.147 & 1 & L2R3 & blend \\
\hline 182 & 1084.559 & -2.321 & 1 & L2P3 & \\
\hline 183 & 1092.194 & -2.225 & 1 & L1R0 & \\
\hline 184 & 1092.732 & -2.395 & 1 & L1R1 & \\
\hline 185 & 1093.955 & -2.310 & 1 & L2P5 & weak \\
\hline 186 & 1094.052 & -2.714 & 1 & L1P1 & \\
\hline 187 & 1094.244 & -2.436 & 1 & L1R2 & \\
\hline 188 & 1096.439 & -2.642 & 1 & L1P2 & \\
\hline 189 & 1096.725 & -2.451 & 1 & L1R3 & blend \\
\hline 190 & 1099.788 & -2.620 & 1 & L1P3 & \\
\hline 191 & 1100.165 & -2.460 & 1 & L1R4 & weak \\
\hline 192 & 1108.128 & -2.762 & 1 & LOR0 & \\
\hline 193 & 1108.634 & -2.932 & 1 & LoR1 & \\
\hline 194 & 1110.063 & -3.250 & 1 & LOP1 & blend \\
\hline 195 & 1110.120 & -2.971 & 1 & LOR2 & blend \\
\hline 196 & 1112.495 & -3.177 & 1 & L0P2 & blend \\
\hline 197 & 1112.584 & -2.991 & 1 & L0R3 & blend \\
\hline 198 & 1115.896 & -3.153 & 1 & LOP3 & \\
\hline
\end{tabular}


Table 3. Table of identified interstellar metal lines. For unresolved doublets and triplets an average wavelength and a calculated effective $\log (g f)$ are given. $\mathrm{VC}$ is the velocity component as given in Table 1

\begin{tabular}{|c|c|c|c|c|c|}
\hline No. & $\lambda[\AA]$ & $\log (g f)$ & $\mathrm{VC}$ & Elem. & Remarks \\
\hline 199 & 919.658 & -2.391 & 1,2 & $\mathrm{O} \mathrm{I}$ & triplet, blend \\
\hline 200 & 924.952 & -2.099 & 1,2 & O I & triplet \\
\hline 201 & 925.442 & -2.752 & 1,2 & O I & comp. 2 ? \\
\hline 202 & 926.212 & -0.359 & 1,2 & $\mathrm{Fe} I I$ & blend \\
\hline 203 & 929.517 & -1.928 & 1,2 & O I & triplet, blend \\
\hline 204 & 930.257 & -2.571 & 1,2 & O I & weak \\
\hline 205 & 936.629 & -1.729 & 1,2 & O I & triplet \\
\hline 206 & 945.191 & -0.565 & 1,2 & C I & weak \\
\hline 207 & 946.978 & -0.230 & 1,2 & S II & blend \\
\hline 208 & 948.686 & -1.492 & 1,2 & O I & triplet, blend \\
\hline 209 & 950.112 & -1.714 & 1 & O I* & doublet, blend \\
\hline 210 & 950.885 & -2.105 & 1,2 & O I & blend \\
\hline 211 & 952.303 & -2.152 & 1,2 & N I & blend \\
\hline 212 & 952.415 & -2.206 & 1,2 & N I & blend \\
\hline 213 & 953.415 & -1.371 & 1,2 & N I & blend \\
\hline 214 & 953.655 & -1.090 & 1,2 & $\mathrm{~N} \mathrm{I}$ & blend \\
\hline 215 & 953.970 & -0.983 & 1,2 & N I & blend \\
\hline 216 & 954.104 & -1.752 & 1,2 & $\mathrm{~N}$ I & blend \\
\hline 217 & 961.041 & -0.457 & 1,2 & P II & weak \\
\hline 218 & 963.801 & 0.164 & 1,2 & P II & blend \\
\hline 219 & 963.990 & -1.134 & 1,2 & N I & comp.2 blend \\
\hline 220 & 964.626 & -1.326 & 1,2 & N I & \\
\hline 221 & 965.041 & -1.634 & 1,2 & N I & blend \\
\hline 222 & 971.738 & -1.131 & 1,2 & O I & triplet \\
\hline 223 & 977.020 & -0.118 & 1 & C III & blend \\
\hline 224 & 1020.699 & -1.248 & 1,2 & $\mathrm{Si}$ II & \\
\hline 225 & 1031.926 & -0.575 & 1,2 & O VI & \\
\hline 226 & 1036.337 & -0.609 & 1,2 & C II & \\
\hline 227 & 1037.018 & -0.308 & 1,2 & $\mathrm{C} \mathrm{II}^{*}$ & blend \\
\hline 228 & 1037.617 & -0.879 & 1,2 & O VI & blend \\
\hline 229 & 1039.230 & -1.337 & 1,2 & O I & \\
\hline 230 & 1048.220 & -0.612 & 1,2 & Ar I & \\
\hline 231 & 1055.262 & -1.097 & 1,2 & Fe II & weak \\
\hline 232 & 1063.176 & -0.222 & 1,2 & $\mathrm{Fe} I I$ & blend \\
\hline 233 & 1063.972 & -1.347 & 1,2 & Fe II & weak \\
\hline 234 & 1066.660 & -1.177 & 1,2 & Ar I & \\
\hline 235 & 1081.875 & -0.854 & 1,2 & $\mathrm{Fe} I I$ & blend \\
\hline 236 & 1083.990 & -0.987 & 1,2 & N II & \\
\hline 237 & 1096.877 & -0.495 & 1,2 & $\mathrm{Fe} I I$ & blend \\
\hline 238 & 1112.048 & -1.040 & 1,2 & $\mathrm{Fe} I I$ & weak, blend \\
\hline 239 & 1121.975 & -0.699 & 1,2 & $\mathrm{Fe}$ II & \\
\hline 240 & 1122.526 & -0.149 & 1,2 & Fe III & \\
\hline 241 & 1125.448 & -0.959 & 1,2 & $\mathrm{Fe} \mathrm{II}$ & \\
\hline 242 & 1133.665 & -1.222 & 1,2 & $\mathrm{Fe} I I$ & \\
\hline 243 & 1134.165 & -1.270 & 1,2 & N I & comp. 1 blend \\
\hline 244 & 1134.415 & -0.969 & 1,2 & N I & comp. 2 blend \\
\hline 245 & 1134.980 & -0.793 & 1,2 & N I & \\
\hline 246 & 1143.226 & -0.876 & 1,2 & $\mathrm{Fe}$ II & \\
\hline 247 & 1144.938 & 0.021 & 1,2 & Fe II & \\
\hline 248 & 1152.818 & -0.627 & 1,2 & P II & \\
\hline 249 & 1190.416 & -0.301 & 1,2 & Si II & \\
\hline
\end{tabular}

Table 3. continued

\begin{tabular}{rrrlll}
\hline No. & $\lambda[\AA]$ & $\log (g f)$ & VC & Elem. & Remarks \\
\hline 250 & 1193.290 & -0.001 & 1,2 & Si II & \\
251 & 1199.550 & -0.275 & 1,2 & N I & \\
252 & 1200.223 & -0.451 & 1,2 & N I & \\
253 & 1200.710 & -0.752 & 1,2 & N I & \\
254 & 1206.500 & 0.223 & 1,2 & Si III & blend \\
255 & 1250.584 & -1.661 & 1,2 & S II & \\
256 & 1253.811 & -1.361 & 1,2 & S II & \\
257 & 1259.519 & -1.187 & 1,2 & S II & \\
258 & 1260.422 & 0.304 & 1,2 & Si II & blend \\
259 & 1260.533 & -0.602 & 1,2 & Fe II & blend \\
260 & 1277.245 & -1.015 & 1 & C I & weak \\
261 & 1302.168 & -0.612 & 1,2 & O I & \\
262 & 1304.370 & -0.531 & 1,2 & Si II & \\
263 & 1334.532 & -0.593 & 1,2 & C II & \\
264 & 1335.690 & -0.292 & 1,2 & C II* & doublet \\
\hline
\end{tabular}

Table 4. Table of the identified interstellar Lyman-series (H I). All lines are doublets with a maximum separation of $5.4 \mathrm{~m} \AA$ (at $\lambda 1216)$. The resulting wavelength and $\log (g f)$ is given. VC is the velocity component as given in Table 1

\begin{tabular}{rrrrrr}
\hline No. & $\lambda[\AA]$ & $\log (g f)$ & VC & $\begin{array}{r}\text { Lyman } \\
\text { Name }\end{array}$ & Remarks \\
\hline 265 & 915.824 & -3.029 & 1 & 14 & \\
266 & 916.429 & -2.938 & 1 & 13 & \\
267 & 917.181 & -2.840 & 1 & 12 & \\
268 & 918.129 & -2.735 & 1 & 11 & \\
269 & 919.351 & -2.620 & 1 & 10 & \\
270 & 920.963 & -2.493 & 1 & 9 & \\
271 & 923.150 & -2.353 & 1 & 8 & \\
272 & 926.226 & -2.196 & 1 & 7 & \\
273 & 930.748 & -2.016 & 1 & 6 & \\
274 & 937.803 & -1.807 & 1 & $\epsilon$ & \\
275 & 949.743 & -1.555 & 1 & $\delta$ & \\
276 & 972.537 & -1.237 & 1 & $\gamma$ & \\
277 & 1025.722 & -0.801 & 1 & $\beta$ & \\
278 & 1215.670 & -0.079 & 1 & $\alpha$ & \\
279 & 1215.670 & -0.079 & 4 & $\alpha$ & geocoronal \\
\hline
\end{tabular}

\subsection{Lyman series lines}

Of the Lyman series 14 lines are detectable, from which the lines below the $\lambda 915.824$ line are not separated, so that this line marks the interstellar Lyman limit towards HD 93521.

\subsection{Stellar lines}

The two NV-lines at $\lambda \lambda 1239 / 1243$ have a pronounced P-Cygni profile. Within the absorption part both lines show two significant narrow absorption components at -270 and $-340 \mathrm{kms}^{-1}$. These narrow absorption components could be an indication for a disk in the wind of HD 93521 (Bjorkman et al. 1994). The same components 
Table 5. Table of identified stellar $\left(^{*}\right)$ and stellar wind $(\mathrm{w})$ lines. For the N III doublets only the stronger of the two lines is listed. VC is the velocity component as given in Table 1

\begin{tabular}{|c|c|c|c|c|c|}
\hline No. & $\lambda[\AA]$ & $\log (g f)$ & $\mathrm{VC}$ & Elem. & Remarks \\
\hline 280 & 977.020 & -0.118 & 3 & $\mathrm{C}$ III $\left(^{*}\right)$ & \\
\hline 281 & 979.832 & -0.248 & 3 & $\left.\mathrm{~N} \mathrm{III}^{*}{ }^{*}\right)$ & doublet \\
\hline 282 & 979.905 & -0.055 & 3 & N III* $\left.{ }^{*}\right)$ & doublet \\
\hline 283 & 989.799 & -0.671 & 3 & $\mathrm{~N} \operatorname{III}\left({ }^{*}\right)$ & \\
\hline 284 & 989.873 & -0.575 & 3 & $\operatorname{SiII}\left({ }^{*}\right)^{\prime}$ & \\
\hline 285 & 1031.926 & -0.575 & 3 & $\mathrm{OVI}\left({ }^{*}\right)$ & P Cygni \\
\hline 286 & 1037.617 & -0.879 & 3 & O VI $\left(^{*}\right)$ & P Cygni \\
\hline 287 & 1062.662 & -1.097 & 3 & $\operatorname{SIV}\left({ }^{*}\right)$ & \\
\hline 288 & 1072.974 & -0.846 & $3,5,6$ & $\mathrm{~S} \mathrm{IV}^{*}\left(^{*}\right)$ & \\
\hline 289 & 1073.516 & -1.800 & 3 & $\mathrm{~S} \mathrm{IV}^{*}\left({ }^{*}\right)$ & \\
\hline 290 & 1117.977 & -0.024 & 3 & $\mathrm{P} V\left({ }^{*}\right)$ & \\
\hline 291 & 1128.008 & -0.329 & 3 & $\mathrm{P} V\left(^{*}\right)$ & \\
\hline 292 & 1174.933 & -0.459 & 3 & C III* $\left.{ }^{*}\right)$ & \\
\hline 293 & 1175.263 & -0.549 & 3 & C III* $\left(^{*}\right)$ & \\
\hline 294 & 1175.590 & -0.679 & 3 & $\mathrm{C} \mathrm{III*}\left(^{*}\right)$ & \\
\hline 295 & 1175.711 & 0.021 & 3 & C III* $\left(^{*}\right)$ & \\
\hline 296 & 1175.987 & -0.549 & 3 & C III* $\left(^{*}\right)$ & \\
\hline 297 & 1176.370 & -0.459 & 3 & $\mathrm{C} \operatorname{III}^{*}\left(^{*}\right)$ & \\
\hline 298 & 1183.032 & -0.591 & 3 & N III* $\left.{ }^{*}\right)$ & doublet \\
\hline 299 & 1184.514 & -0.195 & 3 & N III* $\left(^{*}\right)$ & doublet \\
\hline 300 & 1206.500 & 0.223 & $3,5,6$ & Si III $\left(^{*}\right)$ & \\
\hline 301 & 1238.821 & -0.503 & $3,5,6$ & $\mathrm{~N} V\left({ }^{*}\right)$ & \\
\hline 302 & 1242.804 & -0.806 & $3,5,6$ & $\mathrm{~N} V\left({ }^{*}\right)$ & \\
\hline 303 & 1247.383 & -0.157 & 3 & $\mathrm{C}$ III $\left(^{*}\right)$ & \\
\hline 304 & 1294.545 & -0.037 & 7 & Si III* (w) & \\
\hline 305 & 1296.726 & -0.127 & 7 & Si III* (w) & \\
\hline 306 & 1298.892 & -0.257 & 7 & Si III* (w) & \\
\hline
\end{tabular}

are also visible in the Si IV doublet at $\lambda \lambda 1394 / 1403$, in Si IV $\lambda 1073$ and in Si III $\lambda 1206$.

The strong SiIII $\lambda \lambda 1300$ triplets are seen as wind absorption lines (Massa 1995). They appear at a range between $-60 \mathrm{~km} \mathrm{~s}^{-1}$ and $-100 \mathrm{~km} \mathrm{~s}^{-1}$, whereas Massa reports a value of $-30 \mathrm{~km} \mathrm{~s}^{-1}$. This difference could be due to some long term wind variability.

The comparatively strong stellar absorption at $1085 \AA$ could not be clearly identified, it is possibly a superposition of different lines. A candidate is the He II $\lambda 1084.9$ line, but as the next lower unblended He II line at $\lambda 958.7$ is rather weak, the identification is not sure. There is a Fe II resonance line at $\lambda 1085.0$ with a low $\log (g f)=-2.106$, but also non-resonance lines of Fe II and Fe III are present in this region. So this line probably requires a more detailed analysis.

\section{Conclusions}

The presented ORFEUS II Echelle spectrum of HD 93521 shows an extraordinary rich variety of very sharp interstellar absorption lines, especially in the wavelength region between $900 \AA$ and $1200 \AA$, which was not very well studied before the two ORFEUS missions and for which only now new observation possibilities exist. Particularly
Table 5. continued

\begin{tabular}{rrrlll}
\hline No. & $\lambda[\AA]$ & $\log (g f)$ & VC & Elem. & Remarks \\
\hline 307 & 1298.946 & 0.443 & 7 & Si III* (w) & \\
308 & 1301.149 & -0.127 & 7 & Si III* (w) & \\
309 & 1303.323 & -0.037 & 7 & Si III* (w) & \\
310 & 1393.755 & 0.012 & $3,5,6$ & Si IV (*) & \\
311 & 1402.770 & -0.292 & $3,5,6$ & Si IV (*) & \\
\hline
\end{tabular}

the nearly complete presence of very sharp $(F W H M \approx$ $100 \mathrm{~m} \AA$ ) $\mathrm{H}_{2}$ absorption lines in this spectrum - which will be analysed in detail in a forthcoming paper - makes it well suited as a reference spectrum for interstellar molecular hydrogen. Molecular hydrogen is partially visible in the interstellar high velocity component too. Additionally some stellar lines show narrow absorption components wich are varying in time and which could be an indication for a disk.

Acknowledgements. ORFEUS could only be realized with the support of all our German and American colleagues and collaborators. The authors wish to thank Philipp Richter and Klaas S. de Boer for helpful discussions. The ORFEUS program was supported by DARA grants WE3 OS 8501 and WE2 QV 9304 and NASA grant NAG5-696.

\section{References}

Abgrall H., Roueff E., Launnay F., Roncin J.-Y., Subtil J.-L., 1993, A\&AS 101, 273

Barnstedt J., Kappelmann N., Appenzeller I., et al., 1999, A\&AS 134, 561

Bjorkman J.E., Ignace R., Tripp T.M., Cassinelli J.P., 1994, ApJ 435, 416

Feibelman W.A., Johannson S., 1995, ApJS 100, 405

Grewing M., Boksenberg A., Savage B.D., et al., 1978, Nat 275, 394

Irvine N.J., 1989, ApJ 337, L33

Keenan F.P., Shaw C.R., Bates B., Dufton P.L., Kemp S.N., 1995, MNRAS 272, 599

Kelly R.L., 1968, Atomic Emission Lines Below 2000 Angstroms, Hydrogen Through Argon, Naval Research Laboratory, Washington DC (see also http://cfawww.harvard.edu/amdata/ampdata/kelly/kelly.html)

Kurucz R.L., 1995, CD No. 23, (http://www.pmp.unihannover.de/projekte/kurucz/sekur.html)

Lennon D.J., Dufton P.L., Keenan F.P., Holmgren D.E., 1991, A\&A 246, 175

Massa D., 1995, ApJ 438, 376

Morton D.C., Dinerstein H.L., 1976, ApJ 204, 1

Morton D.C., 1991, ApJS 77, 119

Ramella M., Morossi C., Santin P., 1980, A\&A 90, 146

Savage B.D., Bohlin R.C., Drake J.F., Budich W., 1977, ApJ 216,291

Spitzer L., Fitzpatrick E.L., 1992, ApJ 391, L41

Spitzer L., Fitzpatrick E.L., 1993, ApJ 409, 299

Widmann H., de Boer K.S., Richter P., Krämer G., et al., 1998, A\&A 338, L1

Widmann H., ORFEUS II-Echelle-Spektren, Untersuchungen des heißen galaktischen Halos, Ph.D. Thesis, Universität Tübingen, 1999 


\section{Appendix: Plots of the spectrum of HD 93521}

The following plots show the complete ORFEUS II Echelle spectrum of HD 93521. The numbers shown in squared brackets correspond to the running numbers given in the Tables $2-5$. Stellar lines are marked as $\left(^{*}\right)$, stellar wind lines as (w). Non-resonance lines are additionally marked with an asterisk, *.

The plots show one Echelle order per plot for wavelengths above $1130 \AA$ (Echelle orders 40 to 49), and half an Echelle order per plot for Echelle orders 50 to 61 $(\lambda<1130 \AA)$ which includes all $\mathrm{H}_{2}$-lines. Thus the wavelength scale changes from order to order, but the relative wavelength scale (radial velocity scale) is nearly constant within these two wavelength ranges.

\section{Addendum}

$\mathrm{H}_{2}$-line $\lambda 997.829$ (No. 111) was erroneously identified with two velocity components. The more probable identification for the weaker component however is the $\mathrm{H}_{2}$-line $\lambda 997.640$ W1P5 with $\log (f)=-1.921$. 

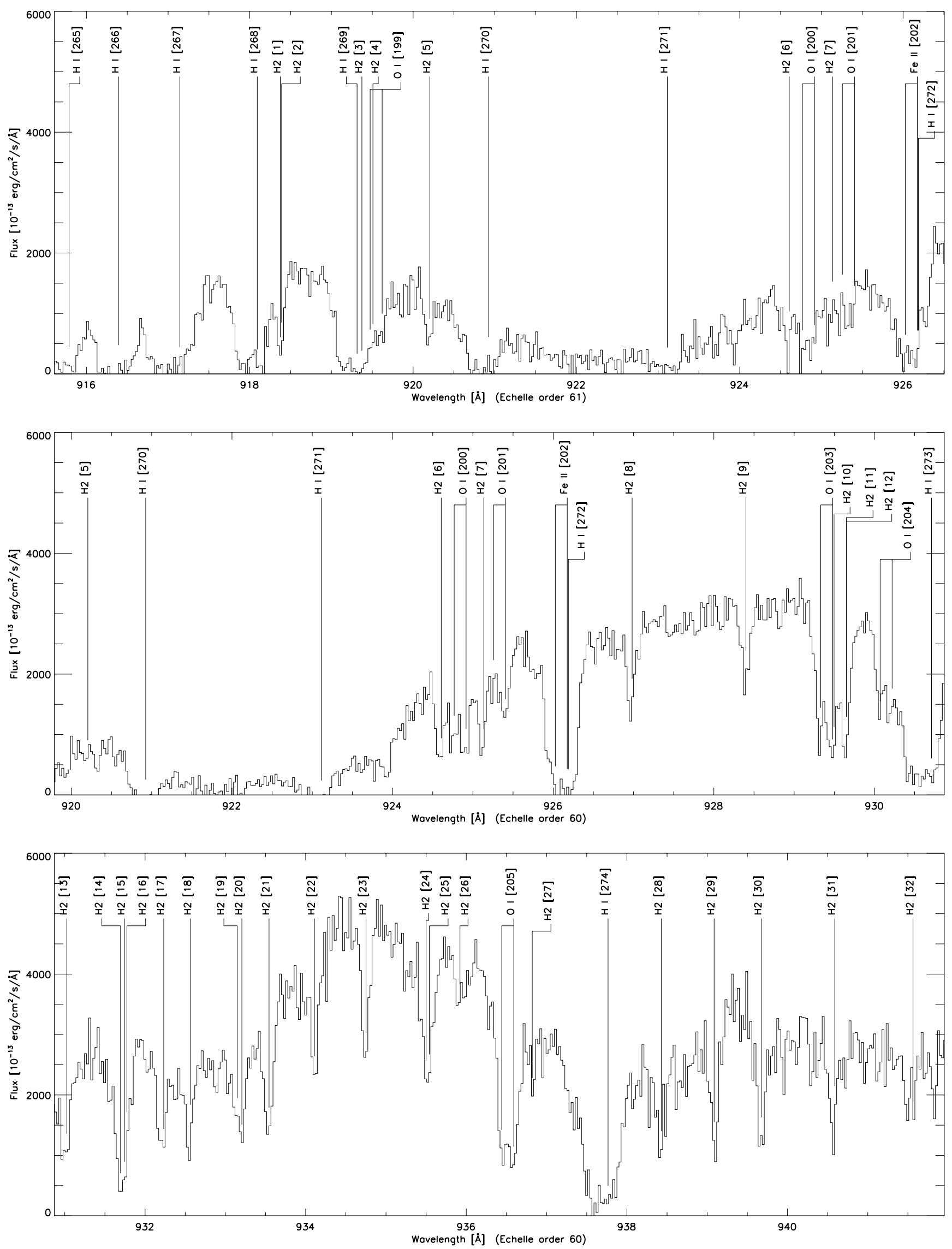

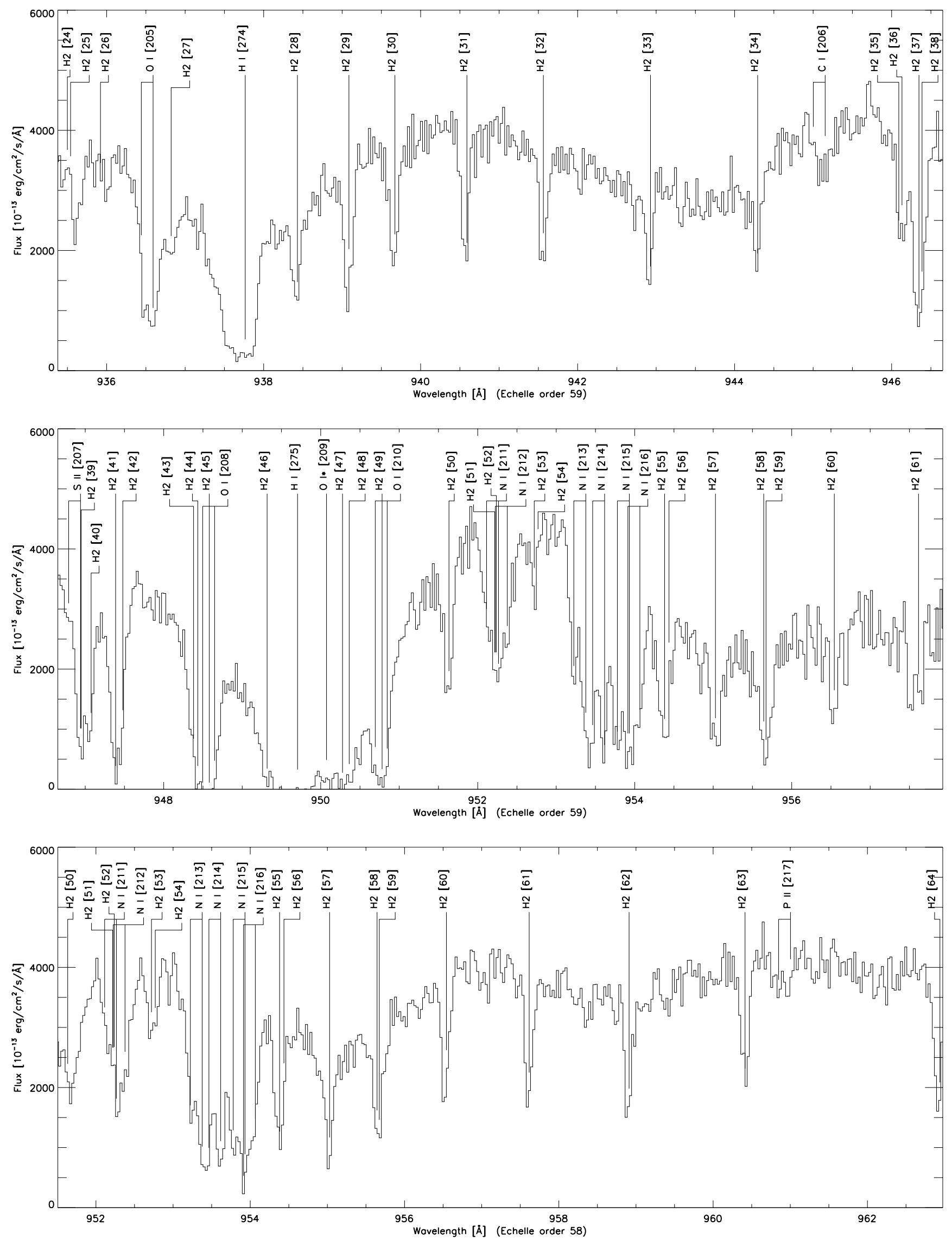

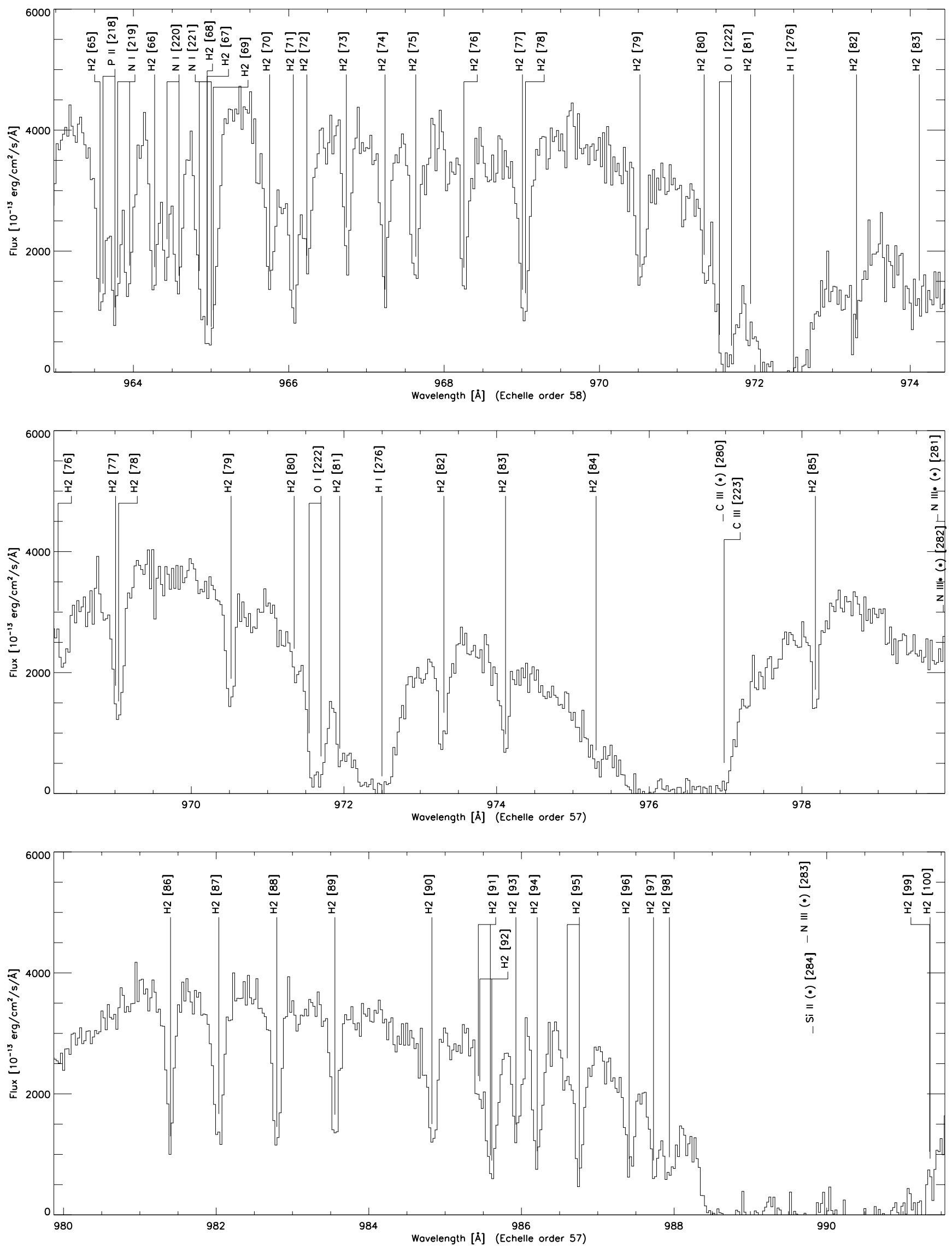

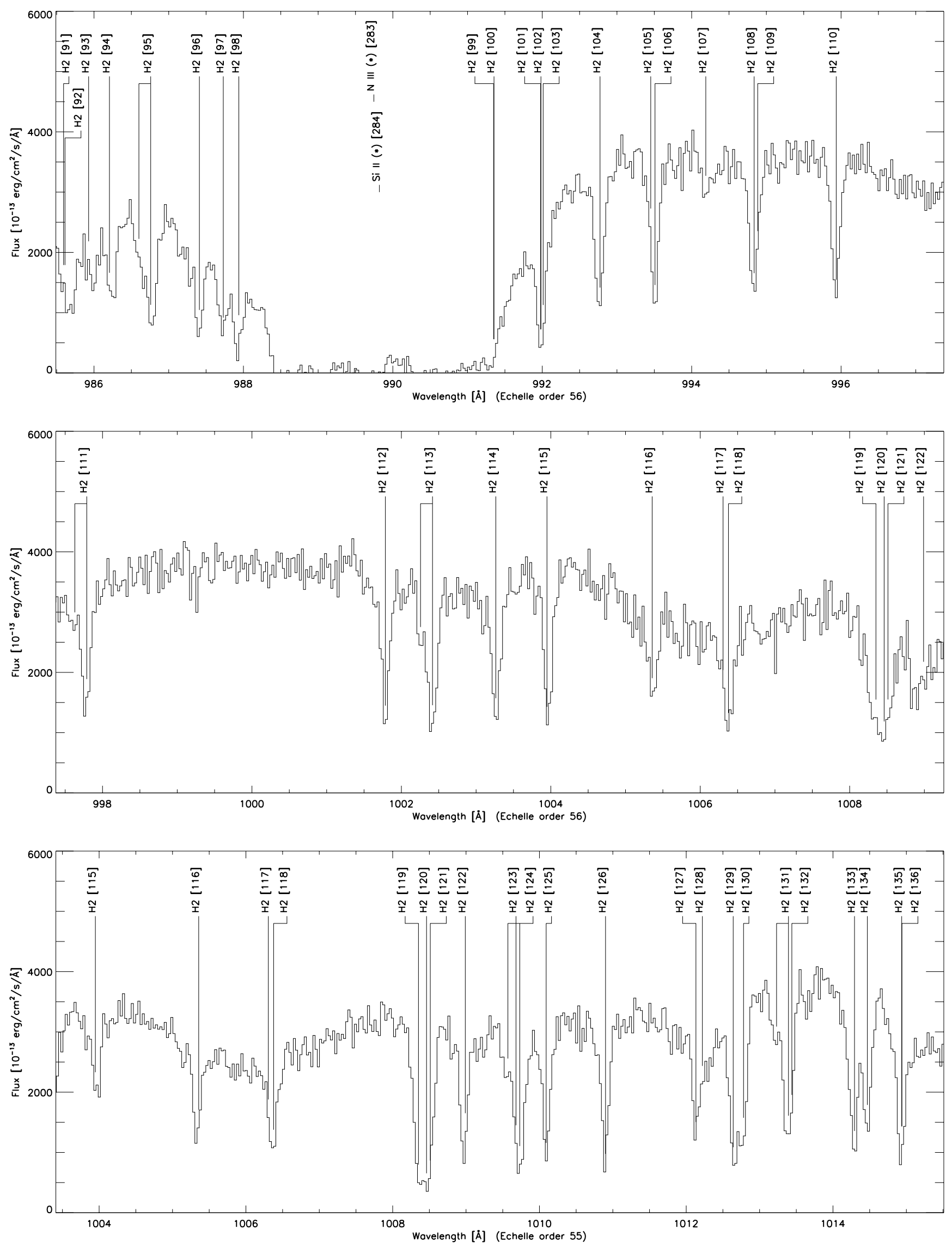

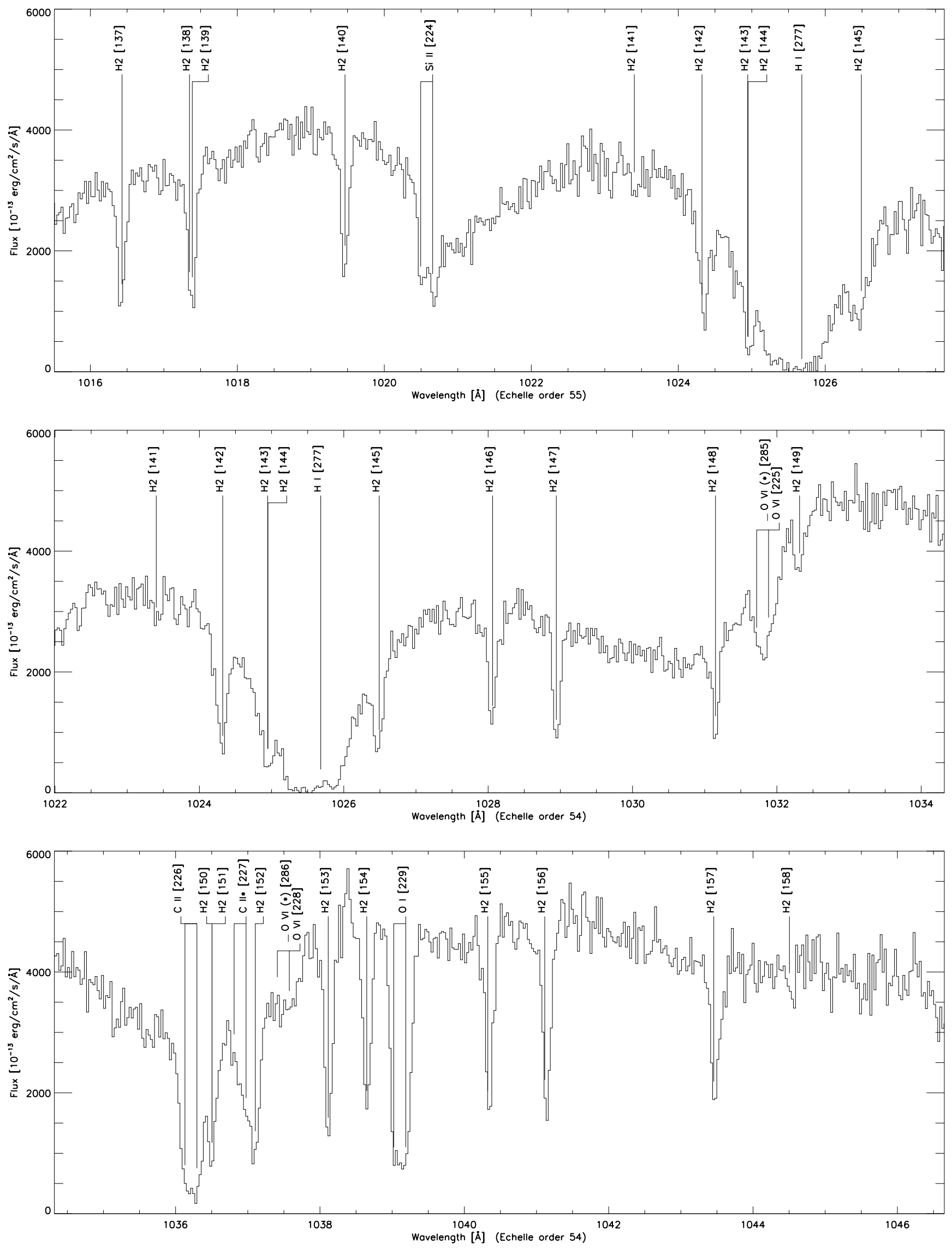

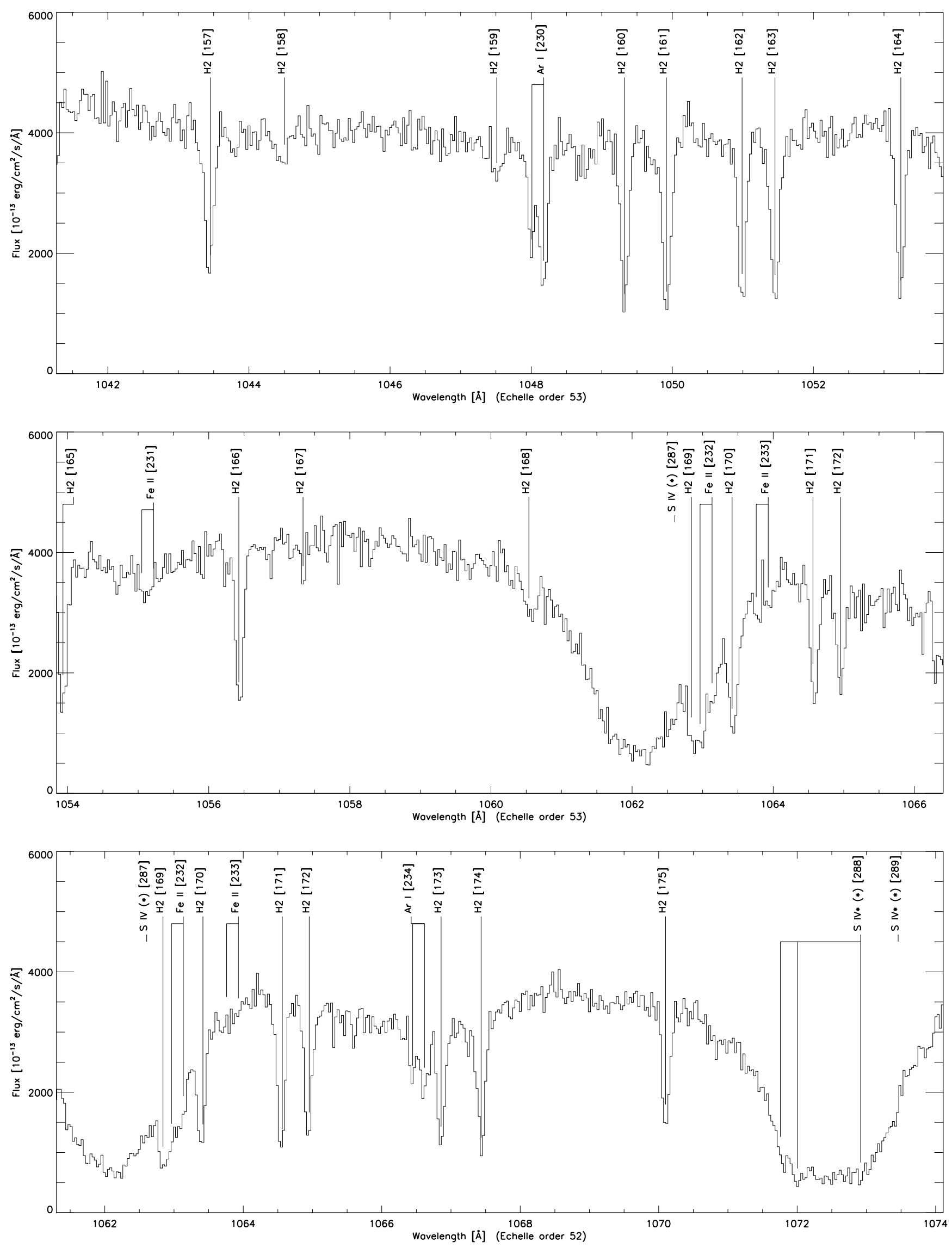

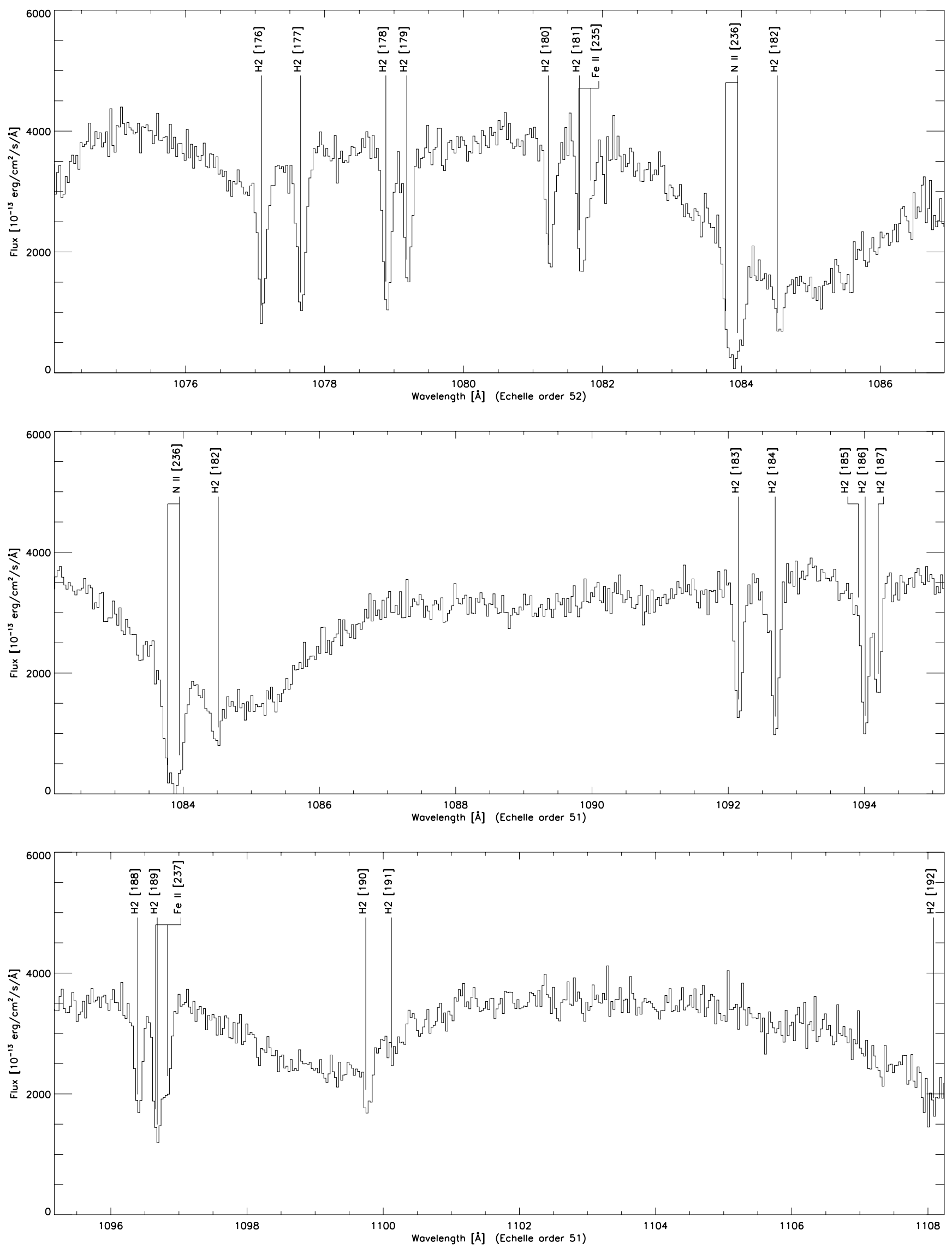

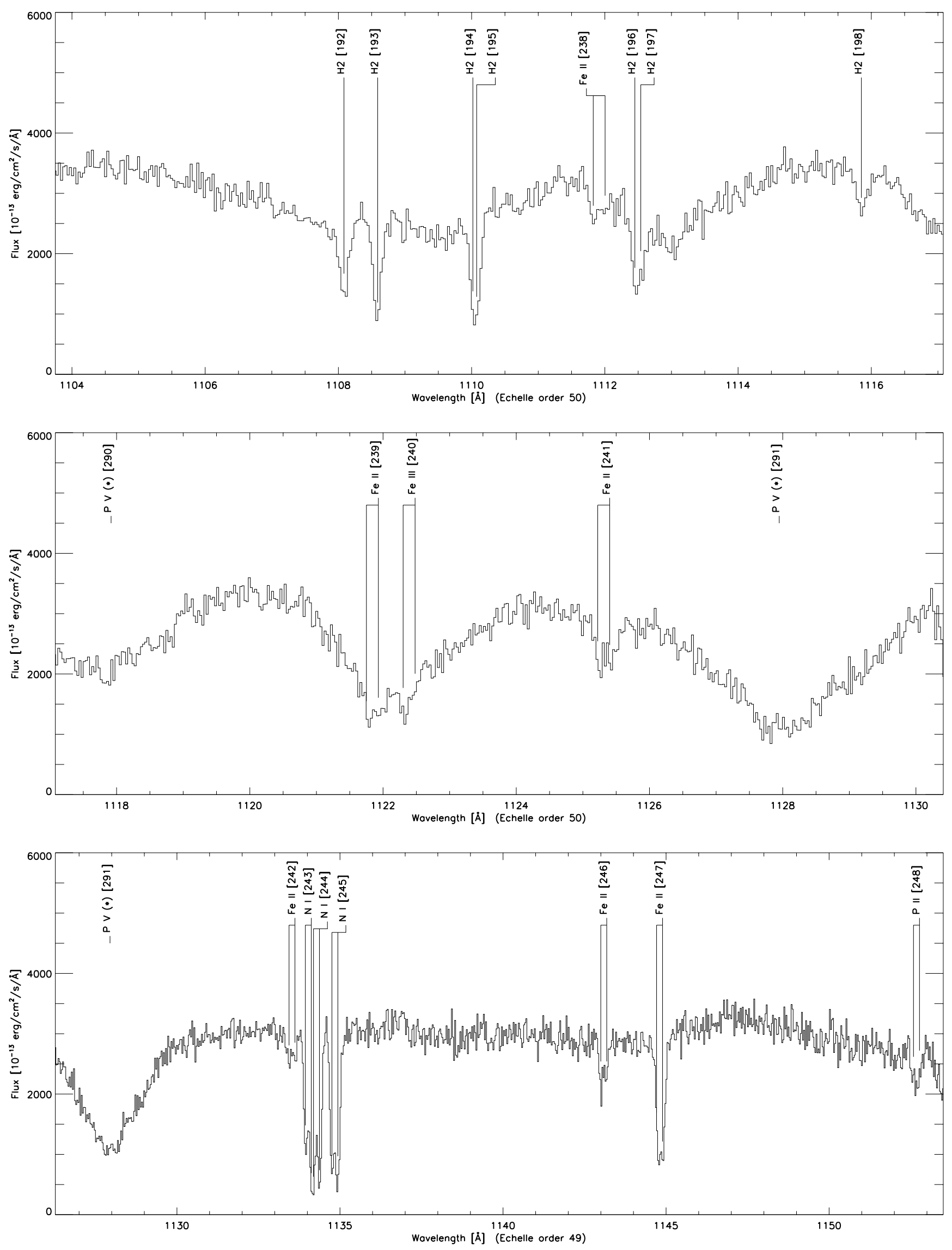

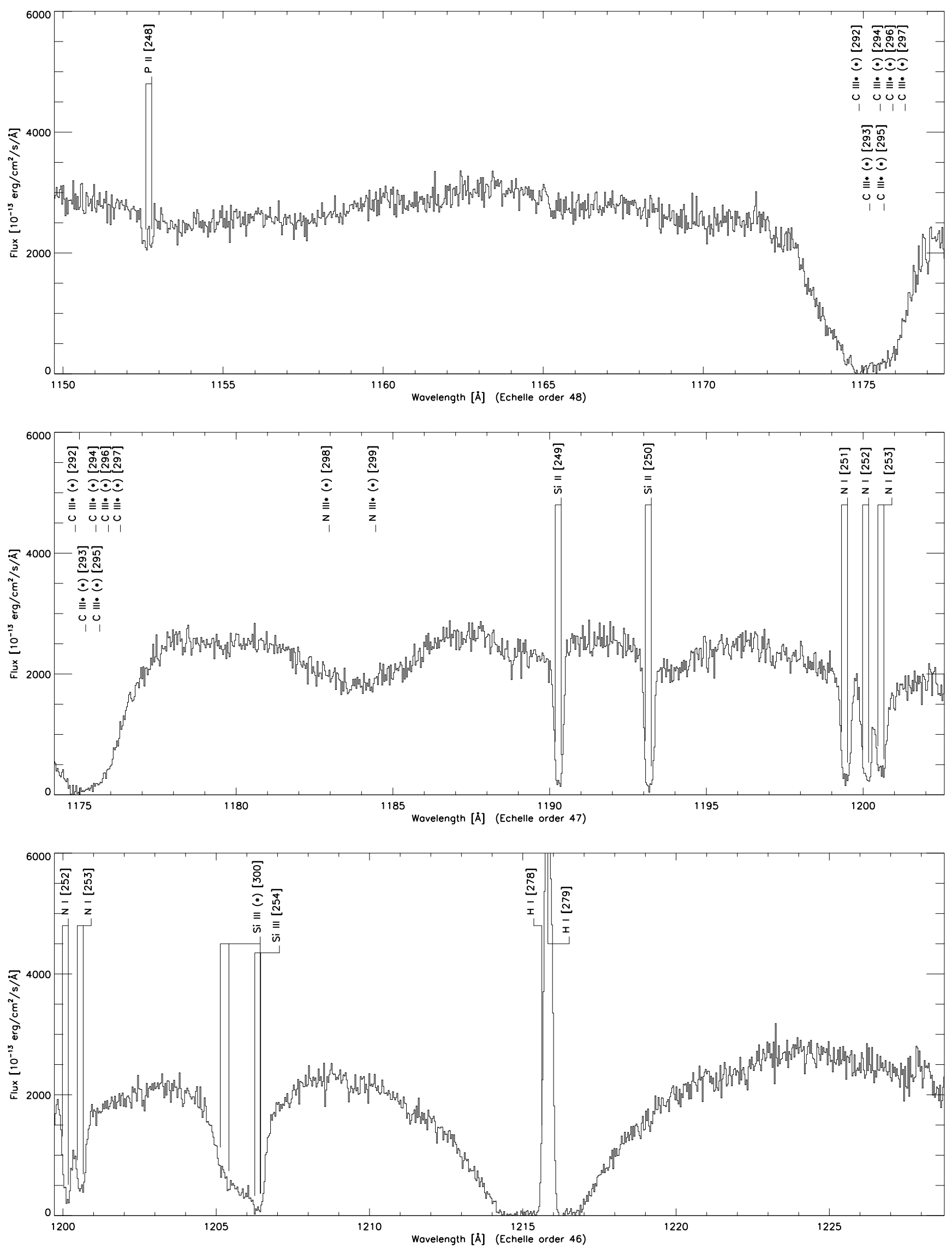

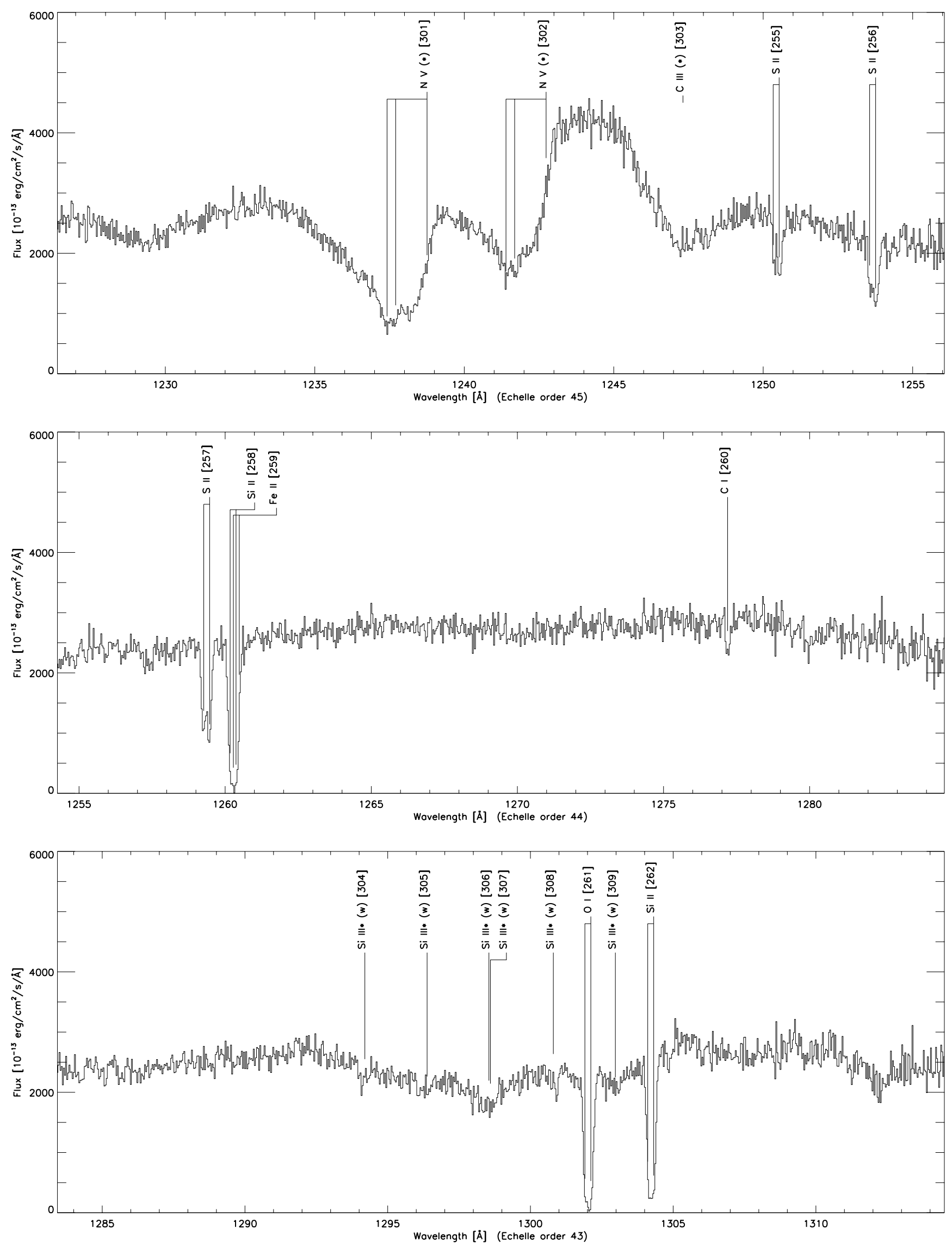

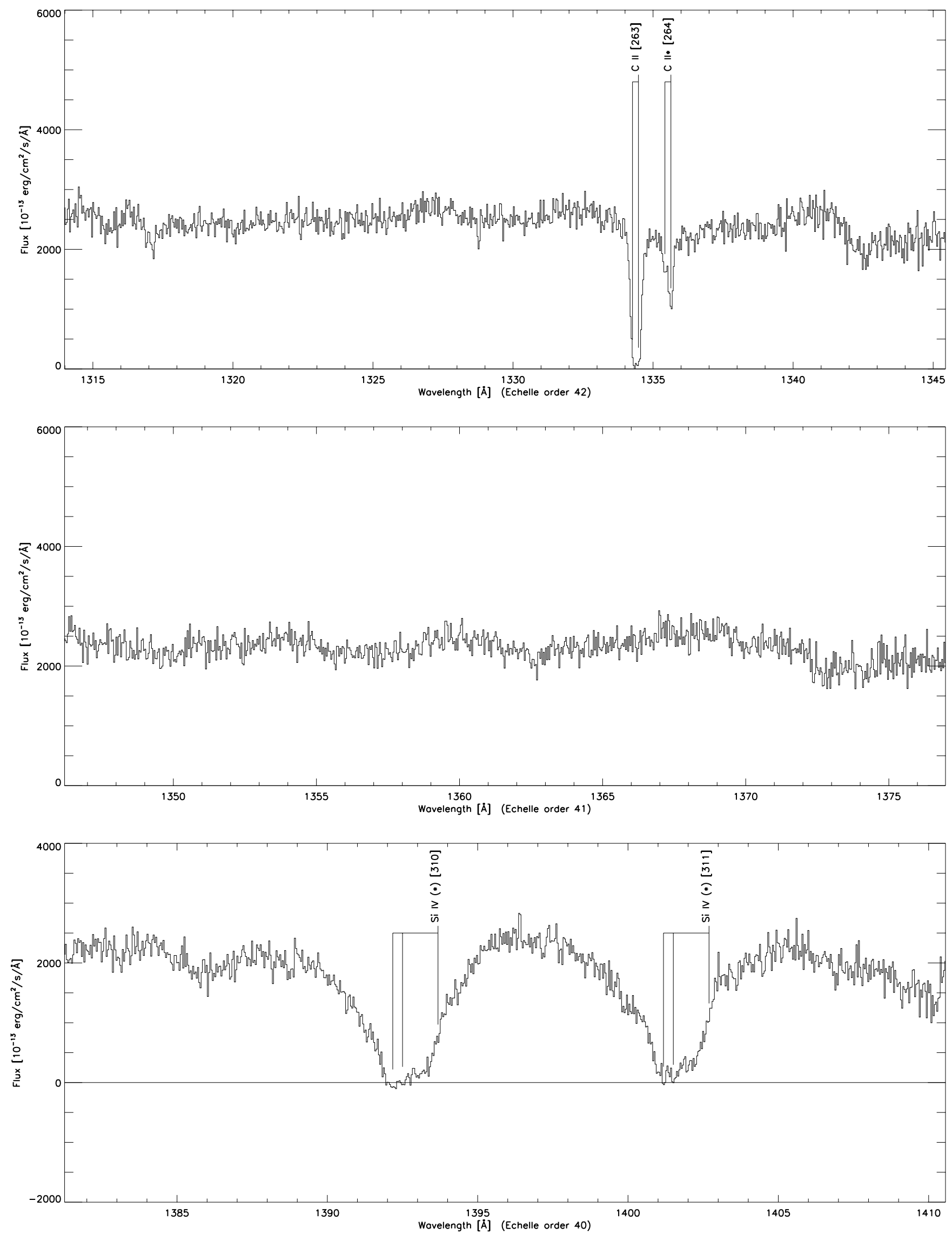